\title{
PERIOD OF BALANCING NUMBERS MODULO PRODUCT OF CONSECUTIVE LUCAS-BALANCING NUMBERS
}

\author{
BIJAN KUMAR PATEL, SHANTA KUMARI SUNANDA, and PRASANTA KUMAR \\ RAY
}

\begin{abstract}
The period of the balancing numbers modulo $m$, denoted by $\pi(m)$, is the least positive integer $l$ such that $\left\{B_{l}, B_{l+1}\right\} \equiv\{0,1\}(\bmod m)$, where $B_{l}$ denotes the $l$-th balancing number. In the present study, we examine the periods of the balancing numbers modulo a product of consecutive Lucas-balancing numbers.
\end{abstract}

MSC 2010. 11B39.

Key words. Balancing number, Lucas-balancing number, periodicity.

\section{REFERENCES}

[1] A. Behera and G.K. Panda, On the square roots of triangular numbers, Fibonacci Quart. 37 (1999), 98-105.

[2] N. Khaochim and P. Pongsriiam, The period modulo product of consecutive Fibonacci numbers, Int. J. Pure Appl. Math., 90 (2014), 335-344.

[3] D. Marques, The order of appearance of product of consecutive Fibonacci numbers, Fibonacci Quart., 50 (2012), 36-43.

[4] D. Marques, The order of appearance of product of consecutive Lucas numbers, Fibonacci Quart., 51 (2013), 36-43.

[5] G.K. Panda, Some fascinating properties of balancing numbers. In: Proceedings of the Eleventh International Conference on Fibonacci Numbers and Their Applications, Congr. Numer., 194 (2009), 185-189.

[6] G.K. Panda and S.S. Rout, Periodicity of balancing numbers, Acta Math. Hungar., 143 (2014), 274-286.

[7] B.K. Patel and P.K. Ray, The period, rank and order of the sequence of balancing numbers modulo m, Math. Rep. (Bucur.), 18 (2016), 395-401.

[8] P.K. Ray, Balancing and cobalancing numbers, PhD Thesis, Department of Mathematics, National Institute of Technology, Rourkela, India, 2009.

[9] P.K. Ray and B.K. Patel, Uniform distribution of the sequence of balancing numbers modulo m, Unif. Distrib. Theory, 11 (2016), 15-21.

[10] P.K. Ray, Some congruences for balancing and Lucas-balancing numbers and their applications, Integers, 14 (2014), \#A8, 1-8.

Received January 31, 2018

Accepted March 24, 2018

DOI: $10.24193 /$ mathcluj.2018.2.10 
International Institute

of Information Technology

Bhubaneswar-751003, India

E-mail: iiit.bijan@gmail.com

E-mail: shanta@iiit-bh.ac.in

Sambalpur University

Burla-768019, India

E-mail: rayprasanta2008@gmail.com 